Twistors and the massive spinning particle

This content has been downloaded from IOPscience. Please scroll down to see the full text.

2016 J. Phys. A: Math. Theor. 49025401

(http://iopscience.iop.org/1751-8121/49/2/025401)

View the table of contents for this issue, or go to the journal homepage for more

Download details:

IP Address: 131.111.184.102

This content was downloaded on 18/12/2015 at 10:18

Please note that terms and conditions apply. 


\title{
Twistors and the massive spinning particle
}

\author{
Luca Mezincescu ${ }^{1}$, Alasdair $\mathrm{J} \mathrm{Routh}^{2}$ and \\ Paul K Townsend ${ }^{2,3}$ \\ ${ }^{1}$ Department of Physics, University of Miami, Coral Gables, FL 33124, USA \\ ${ }^{2}$ Department of Applied Mathematics and Theoretical Physics, Centre for \\ Mathematical Sciences, University of Cambridge, Wilberforce Road, Cambridge, CB3 \\ OWA, UK \\ E-mail: mezincescu@server.physics.miami.edu, A.J.Routh@damtp.cam.ac.uk and \\ P.K.Townsend@damtp.cam.ac.uk
}

Received 26 August 2015, revised 14 October 2015

Accepted for publication 15 October 2015

Published 7 December 2015

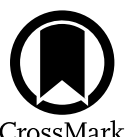

\begin{abstract}
Gauge-invariant twistor variables are found for the massive spinning particle with $\mathcal{N}$-extended local worldline supersymmetry, in spacetime dimensions $D=3,4,6$. The twistor action is manifestly Lorentz invariant but the anticommuting spin variables appear exactly as in the non-relativistic limit. This allows a simple confirmation that the quantum $\mathcal{N}=2$ spinning particle has either spin one or spin zero, and that $\mathcal{N}>2$ is quantum inconsistent for $D=4,6$.
\end{abstract}

Keywords: twistors, spin, supersymmetry

\section{Introduction}

The spacetime dimensions $D=3,4,6$ have the special property that there is an extension of the conformal group, available for all $D$, to a superconformal group [1]. This is relevant to massless supersymmetric field theories, and free field theories of this type arise from quantization of the massless superparticle [2, 3], which is superconformal invariant precisely in dimensions $D=3,4,6$. The superconformal invariance of the massless superparticle action can be made manifest by a formulation $[4,5]$ in which the phase space is parametrized by a supertwistor [6]. Spin-shell constraints then replace the usual mass-shell constraint, ensuring

\footnotetext{
3 Author to whom any correspondence should be addressed.
} 
that the physical phase space dimension is unchanged, and also that the superparticle describes (upon quantization) a supermultiplet of zero super-helicity.

Although the action for a massive particle cannot be conformal invariant, the massive superparticle [7] still has a supertwistor formulation in dimensions $D=3,4,6$ (which we abbreviate to $3 \mathrm{D}$ etc) but now the phase superspace is parametrized by two supertwistors. The necessity of doubling the twistor phase space was initially discovered in the context of the twistor approach to solutions of massive wave equations [8]. One expects to recover such results by covariant quantization of the twistor formulation of corresponding massive particle mechanics models, which is our focus here.

The twistor formulation of at least some massive particle mechanics models may be found indirectly by 'dimensional reduction' of a known twistor formulations of massless particle mechanics models in a higher dimension; in this context, 'dimensional reduction' amounts to the incorporation of an additional constraint on the particle's momentum in the extra dimensions. This naturally leads to a doubled twistor phase space in the lower dimension because (i) a twistor is a spinor of the conformal group [9], which decomposes into a pair of spinors of the lower-dimensional conformal group, and (ii) the conformal invariance in the lower dimension is broken only by the additional momentum constraint, which has no influence on the nature of the phase space.

This construction was first used in [10]: the supertwistor formulation of the massive 4D superparticle was found by reduction of the known supertwistor formulation of the massless $6 \mathrm{D}$ superparticle. In an earlier article, we reviewed and extended the known results on this topic [11], and two of us have recently found (by a direct method) a supertwistor formulation of the massive 6D superparticle [12]. As explained in that work, the combined results for the $D=3,4,6$ massive superparticle fit nicely with the idea $[13,14]$ that properties of supersymmetric theories in spacetime dimensions $D=3,4,6,10$ are related to the division algebras $\mathbb{R}, \mathbb{C}, \mathbb{H}, \mathbb{O}$.

It has been known for a long time [15] that there is also a supertwistor formulation of the 4D massless 'spinning particle' $[16,17]$, which has local worldline supersymmetry rather than global spacetime supersymmetry. The spinning particle action in supertwistor variables is remarkably similar to that of the superparticle but the spin-shell constraints are slightly different, breaking superconformal invariance to conformal invariance and leading to a quantum theory with states of a spin- $1 / 2$ particle rather than a spacetime supermultiplet. This result was generalized in [11] to the $\mathcal{N}$-extended massless 4D spinning particle [18, 19], which describes a particle of spin $\mathcal{N} / 2$, and the results were then used to find analogous results for the massive $3 \mathrm{D}$ spinning particle. However, the constructions underlying these results appear to apply only to a few special cases.

In this paper we present a twistor formulation of the massive spinning particle in $D=3,4,6$, for any $\mathcal{N}$. Our 3D results duplicate those of [11] but our improved construction generalizes to both $4 \mathrm{D}$ and $6 \mathrm{D}$. We say 'twistor' rather than supertwistor because the anticommuting phase-space variables turn out to be different (for $D=4,6$ ) from those of the superparticle. Implicit in our results is a twistor formulation of the massless spinning particle for $D=3,4,6$. We present the details for $D=3$, showing how conformal invariance is recovered in the massless limit. For $D=4$ the analogous final result differs slightly from [15] because the starting point there was the standard form of the massless spinning particle, which differs from what one gets by taking the zero-mass limit of the standard massive spinning particle action.

The main point of our twistor reformulation of massive 'spinning particle' mechanics is that the twistor variables are gauge invariant with respect to local worldline supersymmetry. New gauge invariances are introduced, but not 'fermionic' ones, which means that all 
twistorial anticommuting variables are physical; they appear in the action in exactly the same way that they would in the analogous non-relativistic action! This feature simplifies the determination of some properties of the quantum theory, in particular for the $\mathcal{N}$-extended spinning particle.

For $\mathcal{N}>2$ it is known (and we confirm) that the massive spinning particle model is inconsistent for even $D$ because of a global anomaly. This follows, as pointed out in [20], from a global anomaly exhibited there for the massless $\mathcal{N}>2$ superparticle in odd $D$. This problem can be evaded for $\mathcal{N}=2$ because in this case it is possible to cancel the anomaly by adding a worldline Chern-Simons (WCS) term with half-integral coefficient [20]. We use our results to confirm that the $\mathcal{N}=2$ massive 4D superparticle describes either a spin-zero or a spin-one particle depending on the choice of WCS coefficient.

We should mention here that our initial motivation for considering twistor formulations of massive particle mechanics models was a similarity to twistor formulations of the NambuGoto string [21, 22]. In both cases a doubling of the twistor phase space is needed (compared to a massless particle). The constructions described here for the massive spinning particle may therefore be useful in any future attempt to find a twistor formulation of the spinning string.

We begin with a summary of the $\mathcal{N}$-extended spinning particle action in arbitrary spacetime dimension $D$. We then proceed to its twistor formulation for $D=3,4,6$, dealing sequentially with these dimensions. For $3 \mathrm{D}$ we discuss only the $\mathcal{N}=1$ case; this suffices to introduce the new construction and some generic features of our spinor conventions. For 4D we first discuss the $\mathcal{N}=1$ case and then generalize to $\mathcal{N}>1$, using the results to discuss the quantum theory. For 6D we take over some results of [12] for the bosonic particle, making explicit some conventions implicit in that work, and then present the twistor form of the massive spinning particle. We conclude with a discussion of some general features of our results.

Finally, we include an appendix in which the supertwistor form of the 4D superparticle action is found in the conventions of this paper. This is essentially a more elegant version of previous results but we also keep track of the sign of the energy in the solution to the massshell constraint in order to illustrate an important difference between spinning particles and superparticles.

\section{Spinning particle preliminaries}

For any spacetime dimension, the phase-space action for the minimal massive spinning particle, with $\mathcal{N}=1$ worldline supersymmetry, is

$S=\int \mathrm{d} t\left\{\dot{X}^{m} P_{m}+\frac{\mathrm{i}}{2} \lambda^{m} \dot{\lambda}_{m}+\frac{\mathrm{i}}{2} \xi \dot{\xi}-\frac{1}{2} \mathrm{e}\left(P^{2}+m^{2}\right)+\mathrm{i} \zeta\left(\lambda^{m} P_{m}+m \xi\right)\right\}$.

We use here, and throughout the paper, the Minkowski metric with 'mostly plus' signature. The canonical variables $\left(\lambda^{m}, \xi\right)$ are anticommuting, as is the Lagrange multiplier $\zeta$. The Hamiltonian constraints are both first class, and they generate the gauge transformations

$$
\delta X^{m}=a P^{m}-\mathrm{i} \epsilon \lambda^{m}, \quad \delta \lambda^{m}=P^{m} \epsilon, \quad \delta \xi=m \epsilon,
$$

for commuting parameter $a(t)$ and anticommuting parameter $\epsilon(t)$. The action is invariant if the Lagrange multipliers are assigned the transformations

$$
\delta \mathrm{e}=\dot{a}-2 \mathrm{i} \epsilon \zeta, \quad \delta \zeta=\dot{\epsilon} .
$$

This model describes, upon quantization, a massive spin-1/2 particle [17], the fermionic constraint becoming the Dirac equation when imposed as a physical state condition. Notice 
that the bosonic phase space is spanned by two $D$-vectors subject to one first-class constraint, to which we must add one anticommuting $D$-vector and one anticommuting scalar that are also subject to one first-class constraint. This leads to a physical phase superspace with graded dimension $(2 D-2 \mid D-1)$.

If one takes the $m=0$ limit of the above results then one arrives at the action

$$
S=\int \mathrm{d} t\left\{\dot{X}^{m} P_{m}+\frac{\mathrm{i}}{2} \lambda^{m} \dot{\lambda}_{m}+\frac{\mathrm{i}}{2} \xi \dot{\xi}-\frac{1}{2} \mathrm{e} P^{2}+\mathrm{i} \zeta \lambda^{m} P_{m}\right\}
$$

We could omit the $\xi \dot{\xi}$ term because $\xi$ is now inert under the local worldline supersymmetry, and it has dropped out of the constraints. Indeed, this term is omitted from the standard 'massless spinning particle' action, but omitting it introduces a discontinuity into the massless limit of the massive spinning particle.

The $\mathcal{N}$-extended massive spinning particle is constructed by incorporating more anticommuting variables. Specifically, we make the replacement

$$
\lambda^{m} \rightarrow \lambda_{a}^{m}, \quad \xi \rightarrow \xi_{a}, \quad \zeta \rightarrow \zeta_{a} \quad(a=1, \ldots, \mathcal{N})
$$

and then gauge the resulting $S O(\mathcal{N})$ symmetry by including additional Hamiltonian constraints, imposed by means of a new commuting antisymmetric $S O(\mathcal{N})$-tensor Lagrange multiplier $f_{a b}$. The resulting action is $[18,19]$

$$
\begin{aligned}
S= & \int \mathrm{d} t\left\{\dot{X}^{m} P_{m}+\frac{\mathrm{i}}{2} \lambda_{a} \cdot \dot{\lambda}_{a}+\frac{\mathrm{i}}{2} \xi_{a} \dot{\xi}_{a}-\frac{1}{2} \mathrm{e}\left(P^{2}+m^{2}\right)+\mathrm{i} \zeta_{a}\left(\lambda_{a} \cdot P+m \xi\right)\right. \\
& \left.-\mathrm{i} f_{a b}\left(\lambda_{a} \cdot \lambda_{b}+\xi_{a} \xi_{b}\right)\right\},
\end{aligned}
$$

where summation over the $S O(\mathcal{N})$ indices is implicit. This action has a local $S O(\mathcal{N})$ gauge invariance in addition to its $\mathcal{N}$ local worldline supersymmetries.

The $\mathcal{N}=2$ case is special because then $f_{a b} \propto \epsilon_{a b} f$ with an $S O(2)$ transformation of $f$ that is a total derivative, allowing us to add to the action a term linear in $f$; this is the WCS term. We now have the action

$$
\begin{aligned}
S=\int \mathrm{d} t\left\{\dot{X}^{m} P_{m}+\right. & \frac{\mathrm{i}}{2} \lambda_{a} \cdot \dot{\lambda}_{a}+\frac{\mathrm{i}}{2} \xi_{a} \dot{\xi}_{a}-\frac{1}{2} \mathrm{e}\left(P^{2}+m^{2}\right)+\mathrm{i} \zeta_{a}\left(\lambda_{a} \cdot P+m \xi_{a}\right) \\
& \left.-f\left[\epsilon^{a b}\left(\lambda_{a} \cdot \lambda_{b}+\xi_{a} \xi_{b}\right)-c\right]\right\},
\end{aligned}
$$

where the constant $c$ is the coefficient of the WCS term; it is quantized in the quantum theory with choices that lead to a particle mechanics description of massive $p$-form fields for some integer $p[20]$.

\section{Massive 3D spinning particle}

We shall use a notation in which spacetime vectors are bi-spinors of $\operatorname{Sl}(2 ; \mathbb{R})$. We spell out our conventions here because some important features that carry over to $4 \mathrm{D}$ and $6 \mathrm{D}$ are simpler to discuss for $3 \mathrm{D}$. We start from the real $2 \times 2$ Dirac matrices $^{3}$

$$
\left(\gamma^{0}\right)_{\beta}^{\alpha}=\mathrm{i} \sigma_{2}, \quad\left(\gamma^{1}\right)_{\beta}^{\alpha}=\sigma_{3}, \quad\left(\gamma^{2}\right)_{\beta}^{\alpha}=-\sigma_{1}
$$

3 This differs slightly from the choice made in [11]. 
These matrices satisfy the identities

$$
\left(\gamma^{m}\right)_{\beta}^{\alpha}\left(\gamma_{m}\right)_{\delta}^{\gamma} \equiv 2 \delta_{\delta}^{\alpha} \delta_{\beta}^{\gamma}-\delta_{\beta}^{\alpha} \delta_{\delta}^{\gamma}, \quad\left(\gamma^{m}\right)^{\alpha}{ }_{\beta}\left(\gamma^{n}\right)_{\alpha}^{\beta} \equiv 2 \eta^{m n},
$$

where $\eta$ is the (mostly plus) Minkowski metric. Spinor indices will be raised or lowered by means of the alternating invariant tensor $\varepsilon$ of $\operatorname{Sl}(2 ; \mathbb{R})$, using the conventions

$$
\psi^{\alpha}=\varepsilon^{\alpha \beta} \psi_{\beta}, \quad \psi^{\beta} \varepsilon_{\beta \alpha}=\psi_{\alpha},
$$

for any spinor $\psi$. We define $\varepsilon$ such that

$$
\varepsilon^{\alpha \beta} \varepsilon_{\alpha \gamma}=\delta_{\gamma}^{\beta} \quad\left(\varepsilon^{12}=\varepsilon_{12}=1\right) .
$$

As observed by Howe in the context of $\operatorname{Sl}(2 ; \mathbb{C})$ spinor index conventions [23], these definitions have the advantage that $\varepsilon_{\alpha \beta}$ and $\varepsilon^{\alpha \beta}$ are related by raising/lowering both indices, so that $\varepsilon$ can be consistently interpreted as an $\operatorname{Sl}(2 ; \mathbb{R})$ tensor.

Lowering the indices of the Dirac matrices amounts to a multiplication from the left by $-\mathrm{i} \sigma_{2}$, so that

$$
\gamma_{\alpha \beta}^{m}=\left(1, \sigma_{1}, \sigma_{3}\right)
$$

Notice that these are symmetric. Now, for any Lorentz three-vector $V$, we define

$$
V_{\alpha \beta}=\gamma_{\alpha \beta}^{m} V_{m} \quad \Leftrightarrow \quad V^{m}=-\frac{1}{2} \gamma_{\alpha \beta}^{m} V^{\alpha \beta} .
$$

It follows that

$$
V^{2}=-\frac{1}{2} V^{\alpha \beta} V_{\alpha \beta}=-\operatorname{det} V_{\alpha \beta}
$$

Using these conventions, we find that the $\mathcal{N}=13 \mathrm{D}$ massive spinning particle action is

$$
\begin{aligned}
S=\int \mathrm{d} t\left\{-\frac{1}{2} \dot{X}^{\alpha \beta} P_{\alpha \beta}\right. & -\frac{\mathrm{i}}{4} \lambda^{\alpha \beta} \dot{\lambda}_{\alpha \beta}+\frac{\mathrm{i}}{2} \dot{\xi} \dot{\xi}-\frac{1}{2} \mathrm{e}\left(P^{2}+m^{2}\right) \\
& \left.-\frac{\mathrm{i}}{2} \zeta\left(P^{\alpha \beta} \lambda_{\alpha \beta}-2 m \xi\right)\right\} .
\end{aligned}
$$

The Poisson brackets that follow from this action are

$\left\{X^{\gamma \delta}, P_{\alpha \beta}\right\}_{\mathrm{PB}}=-2 \delta_{\alpha}^{(\gamma} \delta_{\beta}^{\delta)}, \quad\left\{\lambda^{\gamma \delta}, \lambda_{\alpha \beta}\right\}_{\mathrm{PB}}=2 \mathrm{i} \delta_{\alpha}^{(\gamma} \delta_{\beta}^{\delta)}, \quad\{\xi, \xi\}_{\mathrm{PB}}=-\mathrm{i}$.

It should be remembered that the Poisson bracket of two anticommuting variables is symmetric under their interchange. Using these Poisson bracket relations, it may be verified that the constraints are first-class and that they generate the gauge transformations

$$
\delta X^{\alpha \beta}=a P^{\alpha \beta}-\mathrm{i} \epsilon \lambda^{\alpha \beta}, \quad \delta \lambda_{\alpha \beta}=P_{\alpha \beta} \epsilon, \quad \delta \xi=m \epsilon .
$$

In addition to its gauge invariances, the action is manifestly Poincaré invariant. The Noether charges for translations and Lorentz rotations are, respectively

$$
\mathcal{P}_{\alpha \beta}=P_{\alpha \beta}, \quad \mathcal{J}_{\alpha}^{\beta}=X^{\beta \gamma} P_{\alpha \gamma}-\frac{1}{2} \delta_{\alpha}^{\beta} X^{\gamma \delta} P_{\gamma \delta}-\frac{\mathrm{i}}{2} \lambda^{\beta \gamma} \lambda_{\alpha \gamma}
$$

One may construct from these Poincaré charges the Pauli-Lubanski pseudoscalar; in spinor notation this is

$$
\mathcal{W}=\frac{1}{2} \mathcal{P}^{\alpha \beta} \mathcal{J}_{\alpha \beta} .
$$


To pass to the twistor form of the action we first solve the mass-shell constraint by expressing the momentum in terms of a pair of commuting spinors $U_{\alpha}^{I}(I=1,2)$ :

$$
P_{\alpha \beta}=\mp U_{\alpha}^{I} U_{\beta}^{J} \delta_{I J}, \quad \operatorname{det} U=m .
$$

The choice of top sign leads to $P^{0}>0$, i.e. positive energy; we allow for either sign of the energy since, ultimately, both positive and negative energies are needed in relativistic field theory. The constraint on the determinant of the $2 \times 2$ matrix with entries $U_{\alpha}{ }^{I}$ is essentially another version of the mass-shell constraint since $P^{2}=-\operatorname{det} P=-(\operatorname{det} U)^{2}$.

Next, we solve the fermionic constraint by writing $\lambda$ in terms of $\xi$ and two new anticommuting variables in the form of a symmetric and traceless $S O(2)$ tensor $\psi_{I J}$ :

$$
\lambda_{\alpha \beta}=\frac{1}{m} U_{\alpha}^{I} U_{\beta}^{J} \psi_{I J}+\frac{1}{m} P_{\alpha \beta} \xi, \quad \psi_{I J}=\psi_{J I}, \quad \delta^{I J} \psi_{I J}=0 .
$$

Notice that the supersymmetry transformation of $\xi$ now implies that of $\lambda$, so that the new anticommuting variables $\psi_{I J}$ are (like $U$ ) inert under the local worldline supersymmetry.

Substitution for $P$ and $\lambda$ yields the Lagrangian

$$
L=\dot{U}_{\alpha}^{I} W_{I}^{\alpha}+\frac{\mathrm{i}}{4} \psi^{I J} \dot{\psi}_{I J}+\frac{\mathrm{d}}{\mathrm{d} t}(\ldots)
$$

where

$$
W^{\alpha I}=\mp X^{\alpha \beta} U_{\beta}^{I} \mp \frac{\mathrm{i}}{m} U_{K}^{\alpha}\left[\varepsilon^{J K} \psi_{I J} \xi \mp \frac{1}{2} \varepsilon^{J L} \psi_{K L} \psi_{I J}\right] .
$$

From this expression we may derive the identity

$$
0 \equiv \Lambda:=\epsilon_{I J}\left[U_{\alpha}^{I} W^{\alpha J}+\frac{\mathrm{i}}{2} \psi_{I K} \psi_{K J}\right]
$$

It should be appreciated here that (in contrast to the 4D case to follow) there is no significance to the position (up or down) of the $I, J, K, L$ indices since they are raised or lowered using the Kronecker delta.

We see from (3.15) that $W$ is canonically conjugate to $U$ but, as things stand, it is not an independent variable. In order to be able to consider it as an independent variable we must impose $\Lambda=0$ as a constraint by means of a Lagrange multiplier. We must also impose the new mass-shell constraint

$$
0=\varphi:=\operatorname{det} U-m \text {. }
$$

In this way we arrive at the action

$$
S=\int \mathrm{d} t\left\{\dot{U}_{\alpha}^{I} W_{I}^{\alpha}+\frac{\mathrm{i}}{4} \psi^{I J} \dot{\psi}_{I J}-s \Lambda-\rho \varphi\right\},
$$

where $s$ and $\rho$ are the Lagrange multipliers. This action is manifestly $S O(2)$ invariant, and this is a gauge invariance because of the constraint $\Lambda=0$. The new mass-shell constraint function $\varphi$ is the generator for the gauge transformations

$$
\delta W^{\alpha I}=b \epsilon^{I J} U_{J}^{\alpha}, \quad \delta \rho=\dot{b},
$$

for parameter $b(t)$. This transformation is equivalent to a time reparametrization. We do not present the proof, which involves consideration of 'trivial' gauge invariances, because it is very similar to the proof in [12] for an analogous gauge invariance of the twistorial $6 \mathrm{D}$ superparticle. 
In the absence of the constraints, the action (3.19) would be invariant under the infinitedimensional group of canonical transformations of the coordinates of a phase superspace of graded dimension $(8 \mid 3)$, but this is broken by the $\Lambda=0$ constraint to $S p(4 ; \mathbb{R}) \times S O(2)$. Each of the two spinor pairs $Z^{I}=\left(U^{I}, W_{I}\right)$ forms an irreducible 4-plet of $S p(4 ; \mathbb{R})$ and together they form a doublet of $S O(2)$. As $S p(4 ; \mathbb{R})$ is a cover of the $3 \mathrm{D}$ conformal group such that the 4-plet of $\operatorname{Sp}(4 ; \mathbb{R})$ is its spinor representation, this makes $Z^{I}$ a pair of $3 \mathrm{D}$ twistors, so the bosonic phase space is parametrized by a pair of 3D twistors, as claimed. However, the mass-shell constraint $\varphi=0$ breaks $\operatorname{Sp}(4 ; \mathbb{R})$ to $\operatorname{Sl}(2 ; \mathbb{R})$, i.e. to the Lorentz group, as expected for a massive particle.

What we wish to emphasize about the action (3.19) is that there is no trace of the local worldline supersymmetry of the action (3.8) from which we started. The new variables $U$ and $\psi$ are manifestly inert under the local supersymmetry but it is not obvious that $W$ is too. Initially, $W$ was given by the expression (3.16); from this expression we may compute the local supersymmetry transformation of $W$ from the known transformations of $X$ and $\xi$. Using the identity

$$
\varepsilon^{I K} \psi_{K J} \equiv \varepsilon^{J K} \psi_{K I}
$$

one finds that

$$
\delta_{\epsilon} W^{\alpha I}=-\mathrm{i} \epsilon \xi \varepsilon^{I J} U_{J}^{\alpha} .
$$

Although this is non-zero, it has the form of (3.20) with a parameter $b=-\mathrm{i} \epsilon \xi$. Thus, the new variables $(U, V, \psi)$ are gauge invariant with respect to the original gauge transformations modulo a gauge transformation associated to the new mass-shell constraint.

The Pauli-Lubanski pseudoscalar in twistor variables is, after using the mass-shell and spin-shell constraints

$$
\mathcal{W}= \pm m \Sigma, \quad \Sigma=\frac{\mathrm{i}}{4} \epsilon^{I J} \psi_{I K} \psi_{J K}
$$

The two independent components of $\psi_{I J}$ can be traded for a single complex anticommuting variable $\psi$, such that $\{\psi, \bar{\psi}\}_{\mathrm{PB}}=-\mathrm{i}$, by writing

$$
\psi_{11}=-\psi_{22}=\frac{1}{\sqrt{2}}(\psi+\bar{\psi}), \quad \psi_{12}=\psi_{21}=\frac{1}{\sqrt{2} \mathrm{i}}(\psi-\bar{\psi}) .
$$

On passing to the quantum theory, $\psi \rightarrow \hat{\psi}$ and $\bar{\psi} \rightarrow \hat{\psi}^{\dagger}$, we have $\left\{\hat{\psi}, \hat{\psi}^{\dagger}\right\}=1$ (in units for which $\hbar=1$ ) and hence

$$
\hat{\Sigma}=\hat{n}-\frac{1}{2}, \quad \hat{n}=\hat{\psi}^{\dagger} \hat{\psi}
$$

The operator $\hat{n}$ is a fermi number operator with eigenvalues 0,1 . The eigenvalues of the spin operator $\hat{\Sigma}$ are therefore $\pm 1 / 2$. We thus confirm, for $3 \mathrm{D}$, that the $\mathcal{N}=1$ massive spinning particle action describes a particle of spin $1 / 2$. The $\mathcal{N}>1$ case was dealt with in [11].

\subsection{Massless limit}

For the 3D case we shall present an analysis of the $m=0$ limit. Setting $m=0$ in (3.19) changes only the mass-shell constraint, which is now $\operatorname{det} U=0$. This constraint implies that the spinors $\left\{U^{I} ; I=1,2\right\}$ are linearly dependent. Assuming non-zero $U^{1}$, for simplicity of presentation, we then have 


$$
U^{1}=U, \quad U^{2}=\lambda U
$$

for spinor $U(t)$ and scalar $\lambda(t)$. We may now solve the $\Lambda=0$ constraint by setting

$$
W^{1}=W, \quad W^{2}=\lambda W+\kappa U+V,
$$

for spinor $W(t)$, another scalar $\kappa(t)$, and any spinor $V(t)$ such that

$$
U_{\alpha} V^{\alpha}=-2 \bar{\psi} \psi
$$

A solution for $V$ exists because we are assuming non-zero $U$. We may add to $V$ any multiple of $U$ but the solution for $V$ is unique if we consider it to represent the equivalence class for which $V$ and $V^{\prime}$ are identified if they differ by a multiple of $U$.

Gauge invariance of the relation (3.28) requires the following gauge transformation for $V$

$$
\delta V=-\alpha \lambda V
$$

where $\alpha(t)$ is the scalar parameter for $S O(2)$ gauge transformations. Because of the equivalence relation on $V$, it is inert under the transformation (3.20) with parameter $b(t)$. Gauge invariance of the relations (3.26) and (3.27) requires the scalars $(\lambda, \kappa)$ to transform as follows:

$$
\delta \lambda=-\left(1+\lambda^{2}\right) \alpha, \quad \delta \kappa=-2 \alpha \lambda \kappa-b\left(1+\lambda^{2}\right) .
$$

This shows that we may fix the gauge invariances by setting $\lambda=\kappa=0$. At this point the only non-zero independent variables are $(U, W)$ and $\psi$, and the action reduces to

$$
S=\int \mathrm{d} t\left\{\dot{U}_{\alpha} W^{\alpha}+\mathrm{i} \bar{\psi} \dot{\psi}\right\}
$$

This is the twistor action for the massless $3 \mathrm{D}$ spinning particle. It is manifestly $S p(4 ; \mathbb{R})$ invariant, with $(U, W)$ transforming as a 4-plet, i.e. as a $3 \mathrm{D}$ twistor. Notice that the graded dimension of the phase space is $(4 \mid 2)$, which is what we should expect from a comparison with the action (2.4).

\section{Massive 4D spinning particle}

We shall use a notation in which spacetime vectors are bi-spinors of $\operatorname{Sl}(2 ; \mathbb{C})$. Specifically, for any Lorentz four-vector $V$,

$$
V^{m}=-\frac{1}{2} \sigma_{\alpha \dot{\alpha}}^{m} V^{\alpha \dot{\alpha}}, \quad V^{\alpha \dot{\alpha}}=V^{m} \sigma_{m}^{\alpha \dot{\alpha}} .
$$

Here, $\sigma^{m}=\left(\mathbb{I}_{2}, \sigma\right)$ where $\sigma$ is the triplet of $2 \times 2$ Pauli matrices, and

$$
\sigma_{n}^{\alpha \dot{\alpha}}:=\eta_{n m} \varepsilon^{\alpha \beta} \varepsilon^{\dot{\alpha} \dot{\beta}} \sigma_{\beta \dot{\beta}}^{m} .
$$

The $\sigma$ matrices satisfy the relations

$$
\eta_{m n} \sigma_{\alpha \dot{\alpha}}^{m} \sigma_{\beta \dot{\beta}}^{n}=-2 \varepsilon_{\alpha \beta} \varepsilon_{\dot{\alpha} \dot{\beta}}, \quad \sigma_{\alpha \dot{\alpha}}^{m} \sigma_{n}^{\alpha \dot{\alpha}}=-2 \delta_{n}^{m},
$$

where $\eta$ is the Minkowski metric, which we take to have 'mostly plus' signature.

As (4.2) suggests, spinor indices are raised and lowered by means of the $\operatorname{Sl}(2 ; \mathbb{C})$ invariant alternating tensors. As in $3 \mathrm{D}$, we do this according to the convention that, for any spinor $\psi$,

$$
\varepsilon^{\alpha \beta} \psi_{\beta}=\psi^{\alpha}, \quad \varepsilon^{\dot{\alpha} \dot{\beta}} \psi_{\dot{\beta}}=\psi^{\dot{\alpha}} \quad \psi^{\alpha} \varepsilon_{\alpha \beta}=\psi_{\beta}, \quad \psi^{\dot{\alpha}} \varepsilon_{\dot{\alpha} \dot{\beta}}=\psi_{\dot{\beta}}
$$


For any Lorentz vector $V$ we have

$$
\eta^{m n} V_{m} V_{n} \equiv V^{2}=-\frac{1}{2} V^{\alpha \dot{\beta}} V_{\alpha \dot{\beta}}=-\operatorname{det} V
$$

where the last equality follows from the definition of the determinant of the $2 \times 2$ matrix with entries $V_{\alpha \dot{\alpha}}$. The factors here are a reflection of the fact that $\eta^{\alpha \dot{\alpha}, \beta \dot{\beta}} V_{\beta \dot{\beta}}=-2 V^{\alpha \dot{\alpha}}$.

In these spinor conventions, the action for the $\mathcal{N}=1$ spinning particle is

$$
\begin{gathered}
S=\int \mathrm{d} t\left\{-\frac{1}{2} \dot{X}^{\alpha \dot{\alpha}} P_{\alpha \dot{\alpha}}-\frac{\mathrm{i}}{4} \lambda^{\alpha \dot{\alpha}} \dot{\lambda}_{\alpha \dot{\alpha}}+\frac{\mathrm{i}}{2} \xi \dot{\xi}-\frac{1}{2} \mathrm{e}\left(P^{2}+m^{2}\right)\right. \\
\left.-\frac{\mathrm{i}}{2} \zeta\left(P^{\alpha \dot{\alpha}} \lambda_{\alpha \dot{\alpha}}-2 m \xi\right)\right\} .
\end{gathered}
$$

The canonical Poisson brackets are

$$
\left\{X^{\beta \dot{\beta}}, P_{\alpha \dot{\alpha}}\right\}_{\mathrm{PB}}=-2 \delta_{\alpha}^{\beta} \delta_{\dot{\alpha}}^{\dot{\beta}}, \quad\left\{\lambda^{\beta \dot{\beta}}, \lambda_{\alpha \dot{\alpha}}\right\}_{\mathrm{PB}}=2 \mathrm{i} \delta_{\alpha}^{\beta} \delta_{\dot{\alpha}}^{\dot{\beta}}, \quad\{\xi, \xi\}_{\mathrm{PB}}=-\mathrm{i} .
$$

The gauge transformations of the canonical variables are now

$$
\delta X^{\alpha \dot{\alpha}}=a P^{\alpha \dot{\alpha}}-\mathrm{i} \epsilon \lambda^{\alpha \dot{\alpha}}, \quad \delta \lambda_{\alpha \dot{\alpha}}=P_{\alpha \dot{\alpha}} \epsilon, \quad \delta \xi=m \epsilon .
$$

In addition to its gauge invariances, the action is also invariant under the Poincaré isometries of 4D Minkowski space. The corresponding Noether charges are $\mathcal{P}_{\alpha \dot{\alpha}}=P_{\alpha \dot{\alpha}}$ for translations, and

$$
\begin{aligned}
& \mathcal{J}_{\alpha}^{\beta}=\frac{1}{2} X^{\beta \dot{\alpha}} P_{\alpha \dot{\alpha}}-\frac{1}{4} \delta_{\alpha}^{\beta} X^{\gamma \dot{\gamma}} P_{\gamma \dot{\gamma}}-\frac{\mathrm{i}}{4} \lambda^{\beta \dot{\alpha}} \lambda_{\alpha \dot{\alpha}}, \\
& \overline{\mathcal{J}}_{\dot{\alpha}}{ }^{\dot{\beta}}=\frac{1}{2} X^{\alpha \dot{\beta}} P_{\alpha \dot{\alpha}}-\frac{1}{4} \delta_{\dot{\alpha}}^{\dot{\beta}} X^{\gamma \dot{\gamma}} P_{\gamma \dot{\gamma}}-\frac{\mathrm{i}}{4} \lambda^{\alpha \dot{\beta}} \lambda_{\alpha \dot{\alpha}}
\end{aligned}
$$

for Lorentz rotations.

To construct the twistor form of the action, we proceed as in the $3 \mathrm{D}$ case. We first solve the mass-shell constraint by writing $P$ in terms of the $U(2)$ doublet of commuting complex spinors $U_{\alpha}^{I}(I=1,2)$, and their complex conjugates $\bar{U}_{\dot{\alpha} I}$ :

$$
P_{\alpha \dot{\alpha}}=\mp U_{\alpha}^{I} \bar{U}_{\dot{\alpha} I}, \quad|\operatorname{det} U|^{2}=m^{2},
$$

where det $U$ is the determinant is of the complex $2 \times 2$ matrix with entries $U_{\alpha}^{I}$. Observe that $|\operatorname{det} U|^{2}=\operatorname{det} P=-P^{2}$, so the condition $|\operatorname{det} U|^{2}=m^{2}$ needed to solve the original massshell constraint is again a mass-shell constraint, but now expressed in terms of $U$. The choice of the upper sign in (4.10) again corresponds to positive energy.

We can now solve the fermionic constraint by writing $\lambda$ as

$$
\lambda_{\alpha \dot{\alpha}}=\frac{1}{m} U_{\alpha}^{I} U_{\dot{\alpha}}^{J} \psi_{I J}+\frac{1}{m} P_{\alpha \dot{\alpha}} \xi, \quad\left(\psi_{I J}=\psi_{J I}\right)
$$

where the new anticommuting variables $\psi_{I J}$ constitute an $S U(2)$ triplet. As in the $3 \mathrm{D}$ case, the local supersymmetry transformation of $\lambda$ is now implied by that of $\xi$, so that $\psi_{I J}$ is inert. We may raise and lower $S U(2)$ indices with the invariant alternating tensor, which we do using the same conventions as for $\operatorname{Sl}(2 ; \mathbb{C})$ spinor indices. For example

$$
\psi^{I}{ }_{J}=\varepsilon^{I K} \psi_{K J}=\psi^{I K} \varepsilon_{K J},
$$

which are the (anticommuting) entries of a traceless $2 \times 2$ Hermitian matrix. 
Substituting for $P$ and $\lambda$ in the action, we find the new Lagrangian

$$
L=\dot{U}_{\alpha}^{I} W_{I}^{\alpha}+\dot{\bar{U}}_{\dot{\alpha} I} \bar{W}^{\dot{\alpha} I}+\frac{\mathrm{i}}{4} \psi_{J}^{I} \dot{\psi}_{I}^{J}+\frac{\mathrm{d}}{\mathrm{d} t}(\cdots),
$$

where

$$
\begin{aligned}
W_{I}^{\alpha} & =\mp \frac{1}{2} X^{\alpha \dot{\alpha}} \bar{U}_{\dot{\alpha} I} \pm \frac{\mathrm{i}}{2 m^{2}} U^{\alpha J} \operatorname{det} \bar{U}\left(\psi_{I J} \xi \mp \frac{1}{2} \psi_{I}{ }^{L} \psi_{L J}\right), \\
\bar{W}^{\dot{\alpha} I} & =\mp \frac{1}{2} X^{\alpha \dot{\alpha}} U_{\alpha}^{I} \mp \frac{\mathrm{i}}{2 m^{2}} \bar{U}_{J}^{\dot{\alpha}} \operatorname{det} U\left(\psi^{I J} \xi \mp \frac{1}{2} \psi^{I}{ }_{L} \psi^{L J}\right) .
\end{aligned}
$$

Using these expressions, and the new mass-shell constraint

$$
0=\varphi:=|\operatorname{det} U|^{2}-m^{2},
$$

one may derive the identities

$$
\begin{aligned}
& 0 \equiv G:=U_{\alpha}^{I} W_{I}^{\alpha}-\bar{U}_{\dot{\alpha} I} \bar{W}^{\dot{\alpha} I}, \\
& 0 \equiv \Lambda^{I J}:=U^{\alpha{ }^{(I} W_{\alpha}^{J)}}-\bar{U}^{\dot{\alpha}{ }^{(I} \bar{W}_{\dot{\alpha}}^{J}}+\frac{\mathrm{i}}{2} \psi^{I}{ }_{L} \psi^{L J} .
\end{aligned}
$$

Notice that $\Lambda^{I J}=\Lambda^{J I}$ because of the anticommutativity of $\psi_{I J}$. In order to promote the variables $W$ and $\bar{W}$ to the status of independent variables, these constraints must be imposed by Lagrange multipliers, along with the constraint $\varphi=0$.

To simplify the final result, we first trade the anticommuting variables $\psi_{J}^{I}$ for a real anticommuting three-vector $\psi$ by writing

$$
\psi^{I}{ }_{J}=\sigma^{I} \cdot \psi
$$

The new action now takes the form

$$
S=\int \mathrm{d} t\left\{\dot{U}_{\alpha}^{I} W_{I}^{\alpha}+\dot{\bar{U}}_{\dot{\alpha} I} \bar{W}^{\dot{\alpha} I}+\frac{\mathrm{i}}{2} \psi \cdot \dot{\psi}-\ell G-s_{I J} \Lambda^{I J}-\rho \varphi\right\},
$$

where $\ell, s_{I J}=s_{J I}$ and $\rho$ are Lagrange multipliers for the constraints. Only the constraint functions $\Lambda^{I J}$ involve the anticommuting three-vector $\psi$, and they now take the form

$$
\Lambda_{J}^{I}=\Lambda_{\text {(bos) } J}^{I}-\mathrm{i} \sigma_{J}^{I} \cdot \Sigma, \quad \Sigma=-\frac{\mathrm{i}}{2} \psi \times \psi,
$$

where $\Lambda_{\text {(bos) }}$ is the part independent of $\psi$; it can be read off from (4.16).

The bosonic phase space is now parametrized by two pairs of complex two-component spinors $Z^{I}=\left(U^{I}, W_{I}\right)$, and each pair is a complex 4-plet of $\operatorname{Sp}(4 ; \mathbb{C}) \cong U(2,2)$. Since $U(2,2)$ is (neglecting discrete factors) the product of $U(1)$ with the $4 \mathrm{D}$ conformal group, each of the $Z^{I}$ is a 4D twistor. The bosonic phase space is therefore parametrized by a pair of twistors, as for 3D but its real dimension is now $2 \times 8=16$ and the $4 \mathrm{D}$ conformal invariance is broken by the mass-shell constraint. As there are a total of five first-class constraints generating five gauge invariances, the bosonic dimension of the physical phase space is $16-10=6$. There are also three real anticommuting coordinates not subject to any constraint or gauge invariance, so the graded dimension of the physical phase superspace is $(6 \mid 3)$, as it should be.

From the new action (4.18) we may read off the canonical Poisson brackets. These are

$$
\left\{U_{\alpha}^{I}, W_{J}^{\beta}\right\}_{\mathrm{PB}}=\delta_{\alpha}^{\beta} \delta_{J}^{I}, \quad\left\{\bar{U}_{\dot{\alpha} I}, \bar{W}^{\dot{\beta} J}\right\}_{\mathrm{PB}}=\delta_{\dot{\alpha}}^{\dot{\beta}} \delta_{I}^{J},
$$


and, for the components $\psi_{i}(i=1,2,3)$ of $\psi$,

$$
\left\{\psi_{i}, \psi_{j}\right\} \text { РB }=-\mathrm{i} \delta_{i j}
$$

One can verify that the Poisson bracket algebra of the spin-shell constraint functions $\left(G, \Lambda_{J}^{I}\right)$ is $U(2)$. The anticommuting variables contribute only to the spin part of the $S U(2)$ generators, and one may easily check that their Poisson bracket algebra is

$$
\left\{\Sigma_{i}, \Sigma_{j}\right\}_{\mathrm{PB}}=\varepsilon_{i j k} \Sigma_{k} \text {. }
$$

The constraint function $\varphi$ is manifestly $U(2)$ invariant, so it has zero Poisson brackets with the spin-shell constraints. The gauge invariance it generates has the following transformations for parameter $b(t)$ :

$$
\delta_{b} W_{I}^{\alpha}=b U_{I}^{\alpha} \operatorname{det} \bar{U}, \quad \delta_{b} \bar{W}^{\dot{\alpha} I}=-b \bar{U}^{\dot{\alpha} I} \operatorname{det} U, \quad \delta_{b} \rho=\dot{b} .
$$

As for $3 \mathrm{D}$, it is important to take into account this gauge invariance (which is again equivalent to a time reparametrization) when considering how $W$ transforms under the original local supersymmetry. The latter can be deduced by using the transformations of $X$ and $\xi$ in the expressions of (4.14): this gives a $b$-transformation of the above type with $2 m b=\mathrm{i} \epsilon \xi$. Thus the new twistor variables $(U, W, \psi)$ are gauge invariant with respect to all the original local symmetries modulo a gauge transformation generated by the new mass-shell constraint function $\varphi$.

Although we are calling $G=0$ and $\Lambda^{I J}=0$ the spin-shell constraints, their relation to the particle's spin is not obvious because their inclusion in the action leads to the gauging of an apparently internal $U(2)$ symmetry. In fact, the $U(1)$ constraint $G=0$ is not directly related to the particle's spin, but the $S U(2)$ constraint is, as becomes clear when one considers the Pauli-Lubanski spin vector. In $\operatorname{Sl}(2 ; \mathbb{C})$ spinor notation this is

$$
\mathcal{W}_{\alpha \dot{\alpha}}=\mathrm{i}\left(\mathcal{J}_{\alpha}^{\beta} \mathcal{P}_{\beta \dot{\alpha}}-\overline{\mathcal{J}}_{\dot{\alpha}}^{\dot{\beta}} \mathcal{P}_{\alpha \dot{\beta}}\right) \text {. }
$$

In twistor variables, the Lorentz Noether charges are

$$
\begin{aligned}
& \mathcal{J}_{\alpha}^{\beta}=U_{\alpha}^{I} W_{I}^{\beta}-\frac{1}{2} \delta_{\alpha}^{\beta}\left(U_{\gamma}^{K} W_{K}^{\gamma}\right), \\
& \overline{\mathcal{J}}_{\dot{\alpha}}^{\dot{\beta}}=\bar{U}_{\dot{\alpha} I} \bar{W}^{\dot{\beta} I}-\frac{1}{2} \delta_{\dot{\alpha}}^{\dot{\beta}}\left(\bar{U}_{\dot{\gamma} K} \bar{W}^{\dot{\gamma} K}\right) .
\end{aligned}
$$

Notice that there is no longer a contribution from anticommuting variables, as expected from the fact that these are now Lorentz scalars. When these Poincaré Noether charges are substituted into the expression (4.24) one finds that

$$
\mathcal{W}_{\alpha \beta}= \pm \mathrm{i} \Lambda_{I J}^{(\text {bos })} U_{\alpha}^{I} \bar{U}_{\dot{\alpha}}^{J}
$$

where $\Lambda_{\text {(bos) }}$ is $\Lambda$ without the 'fermionic' term. This shows that the bosonic particle has zero spin. For the spinning particle the additional spin term in $\Lambda_{I J}$ is such that, when $\Lambda_{I J}=0$,

$$
\mathcal{W}_{\alpha \dot{\alpha}}=\mp U_{\alpha}^{J} \bar{U}_{\dot{\alpha} I} \quad \sigma_{J}^{I} \cdot \Sigma \Rightarrow \mathcal{W}^{2}=m^{2} \Sigma^{2} .
$$

In the quantum theory, $\Sigma^{2}$ equals $s(s-1)$ for an irreducible massive spin-s representation of the Poincaré group, but to make use of this fact we must first quantize.

To pass to the quantum theory we use Dirac's prescription to replace Poisson brackets of canonical variables by $-i$ times the (anti)commutator of their corresponding operators. This yields the following canonical anticommutation relation for the components of the operator $\hat{\psi}$ : 


$$
\left\{\hat{\psi}_{i}, \hat{\psi}_{j}\right\}=\delta_{i j} \Rightarrow \hat{\psi}=\frac{1}{\sqrt{2}} \tau \Rightarrow \hat{\Sigma}=\frac{1}{2} \tau
$$

where $\tau$ are Pauli matrices. It then follows that

$$
\hat{\Sigma}^{2}=\frac{3}{4} \mathbb{I} \quad \Rightarrow \quad s=\frac{1}{2} .
$$

As expected, the quantum $\mathcal{N}=1$ spinning particle has spin $1 / 2$.

\subsection{Quantum theory for $\mathcal{N} \geqslant 2$}

The twistor formulation of the $\mathcal{N}$-extended spinning particle can be found by following exactly the same procedure already explained for $D=3$. The resulting action is

$$
\begin{aligned}
S & =\int \mathrm{d} t\left\{\dot{U}_{\alpha}^{I} W_{I}^{\alpha}+\dot{\bar{U}}_{\dot{\alpha} I} \bar{W}^{\dot{\alpha} I}+\frac{\mathrm{i}}{2} \psi_{a} \cdot \dot{\psi}_{a}\right. \\
& \left.-\ell G-s_{i j} \Lambda^{I J}-\rho \varphi-f_{a b} \psi_{a} \cdot \psi_{b}\right\},
\end{aligned}
$$

where $\Lambda^{I J}$ is as given in (4.19) but now with

$$
\Sigma=-\frac{\mathrm{i}}{2} \sum_{a=1}^{\mathcal{N}} \psi_{a} \times \psi_{a}
$$

Proceeding as we did for $\mathcal{N}=1$, we now find for $\mathcal{N} \geqslant 2$ that

$$
\hat{\Sigma}=\frac{1}{2}[\tau \otimes \mathbb{I} \otimes \mathbb{I} \otimes \cdots+\mathbb{I} \otimes \tau \otimes \mathbb{I} \otimes+\cdots+\mathbb{I} \otimes \mathbb{I} \otimes \cdots \otimes \tau] .
$$

This acts on a reducible space of dimension $2^{\mathcal{N}}$. However, we still have to consider the $S O(\mathcal{N})$ constraints; there are $\mathcal{N}(\mathcal{N}-1)$ of them. One implies that the state space is annihilated by the operator

$$
\sum_{i=1}^{3} \tau_{i} \otimes \tau_{i} \otimes \mathbb{I} \cdots \otimes \mathbb{I}
$$

However, this operator has eigenvalues 1 and -3 , so there is no state that satisfies the constraint. The theory is quantum inconsistent!

This result is implicit in the conclusion of [20] that the massless spinning particle is inconsistent for $\mathcal{N}>2$ in odd spacetime dimensions ${ }^{4}$ because of a global anomaly (of the general type discussed in [24]). As pointed out in [20], the massive spinning particle in $D$ dimensions can be obtained by a type of dimensional reduction from the massless spinning particle in $D+1$ dimensions, so we should expect the massive $\mathcal{N}>2$ spinning particle to be quantum inconsistent in even spacetime dimensions. This is what we find for $D=4$.

As also pointed out in [20], the global anomaly can be cancelled for $\mathcal{N}=2$ by the WCS term. We shall now recover this result from our twistor formulation of the model. First, we introduce the complex anticommuting triplet

$$
\psi=\frac{1}{\sqrt{2}}\left(\psi_{1}+\mathrm{i} \psi_{2}\right)
$$

\footnotetext{
${ }^{4}$ In even spacetime dimensions it describes a massless particle of $\operatorname{spin} \frac{1}{2} \mathcal{N}$.
} 
This leads to the $\mathcal{N}=2$ action

$$
\begin{gathered}
S=\int \mathrm{d} t\left\{\dot{U}_{\alpha}^{I} W_{I}^{\alpha}+\dot{\bar{U}}_{\dot{\alpha} I} \bar{W}^{\dot{\alpha} I}+\mathrm{i} \bar{\psi} \cdot \dot{\psi}-\ell G-s_{i j} \Lambda^{I J}-\rho \varphi\right. \\
\left.-\frac{1}{4} f[(\bar{\psi} \cdot \psi-\psi \cdot \bar{\psi})+2 c]\right\},
\end{gathered}
$$

where $c$ is the coefficient of the WCS term. Of course, $\bar{\psi} \cdot \psi=-\psi \cdot \bar{\psi}$ in the classical theory but the expression $(\bar{\psi} \cdot \psi-\psi \cdot \bar{\psi})$ yields the standard fermi oscillator operator ordering in the quantum theory.

The canonical anticommutation relations of the operators $\hat{\psi}$ and $\hat{\psi}^{\dagger}$ are

$$
\left\{\hat{\psi}_{i}, \hat{\psi}_{j}^{\dagger}\right\}=\delta_{i j}
$$

The $S O(2)$ constraint therefore reduces to

$$
n_{1}+n_{2}+n_{3}-\frac{3}{2}+c=0,
$$

where $n_{i}$ are the eigenvalues of the fermi number operators $\hat{n}_{i} \equiv \hat{\psi}_{i}^{\dagger} \hat{\psi}_{i}$ (no sum over $i$ ), and we have allowed for the zero point contributions of each of the three fermi oscillators. We now see that

$$
c=\frac{3}{2}-k, \quad k=0,1,2,3 .
$$

For each choice of $k$ we have

$$
\hat{\Sigma}=-\mathrm{i} \hat{\psi} \times \hat{\psi}^{\dagger} \Rightarrow \hat{\Sigma}^{2}=\left(n_{1}-n_{2}\right)^{2}+\left(n_{2}-n_{3}\right)^{2}+\left(n_{3}-n_{1}\right)^{2} \text {. }
$$

For $k=0,3$ (and hence $|c|=\frac{3}{2}$ ) all $n_{i}$ are equal, so there is a single polarization state with $\hat{\Sigma}^{2}=0$. These two cases describe a particle of zero spin. For $k=1,2$ (and hence $|c|=\frac{1}{2}$ ), either one or two of the $n_{i}$ are zero, and both cases give three polarization states with $\hat{\Sigma}^{2}=2$, which implies a particle of spin 1. Since $c$ is non-zero in all cases, we see that the WCS term is crucial to quantum consistency.

\section{Massive 6D spinning particle}

For the $6 \mathrm{D}$ spinning particle, we could use an $\operatorname{Sl}(2 ; \mathbb{H})$ notation for spinors [13] but it is simpler to use an $S U^{*}(4)$ notation $^{5}$. In this notation, Lorentz six-vectors are converted into antisymmetric bi-spinors by means of a set of six antisymmetric $4 \times 4$ matrices $\Sigma^{m}$ which can be chosen such that $(\alpha, \beta, \gamma, \delta=1,2,3,4)$

$$
\Sigma_{m}^{\alpha \beta} \Sigma_{\alpha \beta}^{n}=\delta_{m}^{n}, \quad \Sigma_{\alpha \beta}^{m} \Sigma_{m}^{\gamma \delta}=\delta_{[\alpha}^{\gamma} \delta_{\beta]}^{\delta},
$$

where

$$
\Sigma_{m}^{\alpha \beta}:=\frac{1}{2} \varepsilon^{\alpha \beta \gamma \delta} \sum_{\gamma \delta}^{n} \eta_{m n}
$$

\footnotetext{
5 The $4 \times 4 S U^{*}(4)$ matrices are found from the $2 \times 2 \operatorname{Sl}(2 ; \mathbb{H})$ matrices by using the $2 \times 2$ Pauli matrix
} representation of the algebra of quaternions. 
For this choice ${ }^{6}$ we have, for example

$$
\mathbb{P}_{\alpha \beta}=\Sigma_{\alpha \beta}^{m} P_{m}, \quad P_{m}=\Sigma_{m}^{\alpha \beta} \mathbb{P}_{\alpha \beta},
$$

As suggested by the definition (5.2), we may raise or lower antisymmetric pairs of spinor indices using the $S U^{*}(4)$ invariant alternating tensor. For example

$$
\mathbb{P}^{\alpha \beta}:=\frac{1}{2} \varepsilon^{\alpha \beta \gamma \delta} \mathbb{P}_{\gamma \delta}
$$

We then find, in agreement with $[11,12]$, that that

$$
\mathbb{P}^{\alpha \beta} \mathbb{P}_{\alpha \beta}=P^{2} .
$$

In the above conventions, the action for the $6 \mathrm{D}$ massive spinning particle is

$$
S=\int \mathrm{d} t\left\{\dot{\mathbb{X}}^{\alpha \beta} \mathbb{P}_{\alpha \beta}+\frac{\mathrm{i}}{2} \lambda^{\alpha \beta} \dot{\lambda}_{\alpha \beta}+\frac{\mathrm{i}}{2} \xi \dot{\xi}-\frac{1}{2} \mathrm{e}\left(\mathbb{P}^{2}+m^{2}\right)+\mathrm{i} \zeta\left(\lambda^{\alpha \beta} \mathbb{P}_{\alpha \beta}+m \xi\right)\right\}
$$

The infinitesimal gauge transformations generated by the constraints are

$$
\delta_{\epsilon} X^{\alpha \beta}=-\mathrm{i} \epsilon \lambda^{\alpha \beta}, \quad \delta_{\epsilon} \lambda_{\alpha \beta}=\epsilon \mathbb{P}_{\alpha \beta} \quad \delta_{\epsilon} \xi=m \epsilon .
$$

The Lorentz Noether charges are

$$
\mathcal{J}_{\alpha}^{\beta}=2 \mathbb{P}_{\alpha \gamma} \mathbb{X}^{\beta \gamma}-\frac{1}{2} \delta_{\alpha}^{\beta} \mathbb{P}_{\gamma \delta} \mathbb{X}^{\gamma \delta}+\mathrm{i} \lambda_{\alpha \gamma} \lambda^{\beta \gamma}
$$

To pass to the twistor form of the action, we first solve the mass-shell constraint as in [12] by setting ${ }^{7}$

$$
\mathbb{P}_{\alpha \beta}=\frac{1}{2} \mathbb{U}_{\alpha}^{I} \mathbb{U}_{\beta}^{J} \Omega_{J I}, \quad \operatorname{det} \mathbb{U}=-m^{2},
$$

where $\mathbb{U}$ is a 4-plet $(I=1,2,3,4)$ of $S U^{*}(4)$ spinors, and $\Omega$ is the antisymmetric $U S p(4)$ invariant matrix (normalized such that $\operatorname{det} \Omega=1$ ). We use $\Omega$ to raise and lower indices using the same conventions that we used previously for $\operatorname{Sl}(2 ; \mathbb{R})$ and $\operatorname{Sl}(2 ; \mathbb{C})$. To verify that the mass-shell constraint is solved, one needs the identity $3 \Omega_{I[J} \Omega_{K L]} \equiv \epsilon_{I J K L}$, where $\epsilon_{I J K L}$ is the invariant alternating tensor of $U S p(4)$. The invariant tensor $\epsilon^{I J K L}$ is then defined by raising indices, which implies that $\epsilon^{1234}=1$ given (as we assume) that $\epsilon_{1234}=1$. As for the 3D and $4 \mathrm{D}$ cases, the constraint on the determinant of $U$ can be viewed as a new mass-shell constraint.

Before proceeding it is convenient to define

$$
\mathbb{V}_{I}^{\alpha}=\frac{1}{6 m} \epsilon_{I J K L} \epsilon^{\alpha \beta \gamma \delta} \mathbb{U}_{\beta}^{J} \mathbb{U}_{\gamma}^{K} \mathbb{U}_{\delta}^{L}
$$

This is a new $U S p(4) 4$-plet of commuting $S U^{*}(4)$ spinors of opposite chirality to $\mathbb{U}$, and the two are inverses of each other, up to factors, since

$$
\mathbb{V}_{I}^{\alpha} \mathbb{U}_{\alpha}^{J}=-m \delta_{I}^{J}, \quad \mathbb{V}_{I}^{\alpha} \mathbb{U}_{\beta}^{I}=-m \delta_{\beta}^{\alpha} \quad\left(\operatorname{det} \mathbb{U}=-m^{2}\right) .
$$

\footnotetext{
6 Our choice of factors differs from those of [25], where other aspects of the $S U^{*}(4)$ spinor notation are explained. 7 The sign of the energy now depends on the choice of the USp(4)-invariant tensor $\Omega$. Also, notice the sign of det $\mathbb{U}$ on the mass shell.
} 
It can be shown, again on the surface $\operatorname{det} \mathbb{U}=-m^{2}$, that [12]

$$
\mathbb{P}^{\alpha \beta}=-\frac{1}{2} \mathbb{V}_{I}^{\alpha} \mathbb{V}_{J}^{\beta} \Omega^{J I}, \quad\left(\operatorname{det} \mathbb{U}=-m^{2}\right) .
$$

Next, we solve the fermionic constraint by setting

$$
\lambda_{\alpha \beta}=\frac{1}{\sqrt{2} m} \mathbb{U}_{\alpha}^{I} \mathbb{U}_{\beta}^{J} \psi_{I J}+\frac{1}{m} \mathbb{P}_{\alpha \beta} \xi
$$

where $\psi_{I J}$ is antisymmetric and $\Omega$-tracefree, and hence has five independent components. As for the $3 \mathrm{D}$ and $4 \mathrm{D}$ cases, the supersymmetry transformation of $\lambda$ is now implied by that of $\xi$, so that the new anticommuting variables $\psi$ are inert. A useful alternative, but equivalent, expression for $\lambda$ is

$$
\lambda^{\alpha \beta}=\frac{1}{\sqrt{2} m} \mathbb{V}_{I}^{\alpha} \mathbb{V}_{J}^{\beta} \psi^{I J}+\frac{1}{m} \mathbb{P}^{\alpha \beta} \xi .
$$

To prove equivalence of this expression to (5.13) one needs the relation

$$
\psi^{I J}=-\frac{1}{2} \epsilon^{I J K L} \psi_{K L}
$$

The left-hand side is defined by raising indices with $\Omega$. To show that this equals the righthand side one uses the identity $\epsilon^{I J K L}=\Omega^{I[J} \Omega^{K L]}$ and the fact that $\psi_{I J}$ is both antisymmetric and $\Omega$-traceless ${ }^{8}$.

Substituting for $\mathbb{P}$ and $\lambda$, one finds that

$$
\dot{\mathbb{X}}^{\alpha \beta} \mathbb{P}_{\alpha \beta}+\frac{\mathrm{i}}{2} \lambda_{\alpha \beta} \dot{\lambda}^{\alpha \beta}+\frac{\mathrm{i}}{2} \dot{\xi}=\dot{\mathbb{U}}_{\alpha}^{I} \mathbb{W}_{I}^{\alpha}+\frac{\mathrm{i}}{4} \psi^{I J} \dot{\psi}_{I J}+\frac{\mathrm{d}}{\mathrm{d} t}(\ldots),
$$

where $^{9}$

$$
W_{I}^{\alpha}=-\mathbb{X}^{\alpha \beta} U_{\beta}^{J} \Omega_{J I}-\frac{\mathrm{i}}{\sqrt{2} m} \mathbb{V}_{K}^{\alpha} \psi^{K}{ }_{I} \xi-\frac{\mathrm{i}}{2 m} \mathbb{V}_{K}^{\alpha} \psi^{K J} \psi_{I J}
$$

This expression implies the identity

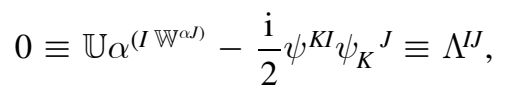

which becomes a constraint imposed by a Lagrange multiplier in the twistor form of the action in which $\mathbb{W}$ is an independent variable. This action is

$$
S=\int \mathrm{d} t\left\{\dot{\mathbb{U}}_{\alpha}^{I} \mathbb{W}_{I}^{\alpha}+\frac{\mathrm{i}}{4} \psi_{I J} \dot{\psi}^{I J}-s_{I J} \Lambda^{I J}-\rho \varphi\right\} .
$$

As anticipated, the only surviving anticommuting phase space variables are the five independent components of $\psi_{I J}$.

The bosonic phase space is now parametrized by the spinor pair $\left(U^{I}, W_{I}\right)$, with each spinor in the $(4,4)$ representation of $S U^{*}(4) \times U S p(4)$; equivalently, each spinor is a pair of $\operatorname{Sl}(2 ; \mathbb{H})$ spinors, and the spinor pair $\left(U^{I}, W_{I}\right)$ is equivalent to a pair of four-component quaternionic spinors in the $(4,2)$ representation of $\operatorname{Sp}(4 ; \mathbb{H}) \times U(2 ; \mathbb{H})$. A single four-

${ }^{8}$ It is not consistent to use the alternating invariant tensor of $U S p(4)$ to raise or lower antisymmetric pairs of $U S p(4)$ spinor indices because a different sign would then be needed to apply this to $\Omega$ itself, as follows from identity $\Omega^{I J} \equiv \frac{1}{2} \epsilon^{I J K L} \Omega_{K L}$

9 We choose the overall sign of $\mathbb{W}$ to be opposite to that chosen in [12] so that the form of the action is similar to the $3 \mathrm{D}$ and $4 \mathrm{D}$ cases deduced in previous sections. 
component quaternionic 4-plet of $\operatorname{Sp}(4 ; \mathbb{H})$ is a spinor of the $6 \mathrm{D}$ conformal group, and hence a $6 \mathrm{D}$ twistor, so the bosonic phase space of the massive $6 \mathrm{D}$ particle described by the action (5.19) is parametrized by a pair of twistors, exactly as we found earlier for $D=3,4$. The real dimension of this space is now $4 \times 8=32$ but these variables are subject to $10+1=11$ first class constraints, which generate 11 gauge invariances, so the physical bosonic dimension of phase space is 10 . There are also five real anticommuting variables, not subject to any constraints, so the graded real dimension of the physical phase superspace is $(10 \mid 5)$, as expected from our starting point.

The new mass-shell constraint $\varphi=0$ is associated with the following gauge invariance with parameter $b(t)$ :

$$
\delta_{b} \mathbb{W}_{\alpha}^{I}=-m b \mathbb{V}_{I}^{\alpha}, \quad \delta_{b} \rho=\dot{b} .
$$

As shown in [12], this is equivalent to a time reparametrization, and as in the $3 \mathrm{D}$ and $4 \mathrm{D}$ cases, a $b$-gauge transformation of $\mathbb{W}$ is induced by a local worldline supersymmetry transformation of $\mathbb{X}$ and $\xi$ in the expression (5.17). Specifically, one finds that

$$
\delta_{\epsilon} \mathbb{W}_{I}^{\alpha}=\mathrm{i} \epsilon \xi V_{I}^{\alpha} \text {. }
$$

The twistor variables are therefore gauge-invariant under the original gauge transformations modulo a $b$-gauge transformation with parameter $b=-\mathrm{i} \epsilon \xi / m$.

We may simplify the action (5.19) by writing

$$
\psi_{I J}=\frac{1}{2}\left(\gamma^{a}\right)_{I J} \psi_{a}
$$

where $\psi_{a}$ is an anti-commuting five-vector, and $\left(\gamma^{a}\right)_{I}^{J}(a=1, \ldots, 5)$ are the five $4 \times 4$ Spin(5) Dirac matrices satisfying

$$
\left\{\gamma^{a}, \gamma^{b}\right\}=2 \delta^{a b}
$$

For the choice $\Omega=\mathbb{I}_{2} \otimes \mathrm{i} \sigma_{2}$, a basis for these matrices is

$$
\gamma^{a}=\left\{\sigma_{2} \otimes \sigma_{1}, \sigma_{2} \otimes \sigma_{2}, \sigma_{2} \otimes \sigma_{3}, \sigma_{1} \otimes \mathbb{I}_{2}, \sigma_{3} \otimes \mathbb{I}_{2}\right\},
$$

and the five antisymmetric matrices with entries $\gamma_{I J}^{a}$ are $\gamma^{a} \Omega$. The action becomes

$$
S=\int \mathrm{d} t\left\{\dot{U}_{\alpha}^{I} \mathbb{W}_{I}^{\alpha}+\frac{\mathrm{i}}{4} \psi_{a} \dot{\psi}_{a}-s_{I J} \Lambda^{I J}-\rho \varphi\right\},
$$

where now

$$
\Lambda^{I J}=\mathbb{U}^{\left(I_{\alpha} \Omega^{J)}\right.} K \mathbb{W}_{K}^{\alpha}+\frac{\mathrm{i}}{8}\left(\gamma^{a b}\right)^{I J} \psi_{a} \psi_{b} \quad\left(\gamma^{a b}=\gamma^{[a} \gamma^{b]}\right) .
$$

The Lorentz Noether charge in the twistor variables are

$$
\mathcal{J}_{\alpha}^{\beta}=\mathbb{U}_{\alpha}^{I} \mathbb{W}_{I}^{\beta}-\frac{1}{4} \delta_{\alpha}^{\beta}\left(\mathbb{U}_{\gamma}^{I} W_{I}^{\gamma}\right)
$$

As expected, there is no fermion bilinear term because the anticommuting variables are Lorentz scalars.

In the quantum theory, the Poisson bracket relations of the anticommuting variables $\psi_{a}$ become the canonical anticommutation relations

$$
\left\{\hat{\psi}_{a}, \hat{\psi}_{b}\right\}=2 \delta_{a b} \Rightarrow \hat{\psi}_{a}=\Gamma_{a}
$$


where $\Gamma_{a}$ are another set of Spin(5) Dirac matrices. This is exactly what one finds in the nonrelativistic limit for a particle of spin 1/2 in five-dimensional Euclidean space, so we confirm that the quantum $\mathcal{N}=16 \mathrm{D}$ massive spinning particle has spin $1 / 2$.

The twistor form of the 6D massive spinning particle action for $\mathcal{N}>1$ can be obtained exactly in the way described earlier for $D=4$. The $\mathcal{N}=2$ case is the one of most interest because the $\mathcal{N}>2$ cases are inconsistent as quantum theories. We pass over the details since the the end results are known from the work of [20]. The procedure is similar to that already described for $4 \mathrm{D}$ but the description of the results obtained involves consideration of the $6 \mathrm{D}$ Pauli-Lubanski tensors given in [12], which goes beyond the scope of this paper.

\section{Discussion}

In this paper we have shown how the massive 'spinning particle' (with local worldline supersymmetry) may be reformulated in twistor variables for spacetime dimension $D=3,4,6$. Our results duplicate those of [11] for $D=3$ but our new construction generalizes directly to $D=4,6$.

A feature of the twistorial action is that the anticommuting spin variables appear exactly as they do in the non-relativistic limit! This is possible because the twistor variables are invariant under the local supersymmetry of the original action. This is also true for the massless spinning particle but the results are more striking for non-zero mass, partly because it is only in this case that one can consider a non-relativistic limit, and partly because the spinshell group (which coincides, or 'almost coincides' with Wigner's little group) is larger for massive particles.

It is also true that the original variables are invariant under the gauge transformations that act on the twistor variables. In this sense, the two formulations are dual, sharing physical content but differing in the extra variables used to ensure manifest Lorentz invariance. Actually, for massive particles both formulations share a common gauge invariance, generated by a Hamiltonian constraint, equivalent in both formulations to a time reparametrization. The mass-shell constraint in one formulation is simply exchanged for a mass-shell constraint in the other. Invariance of one set of variables with respect to the gauge invariances of the other must therefore be understood to be 'modulo' a time-reparametrization invariance.

Because of the simple non-relativistic nature of the 'fermionic' terms in the twistorial version of the massive spinning particle action, the analysis of the implications for the quantum theory is simplified. We illustrated this fact by an analysis of the 4D $\mathcal{N}$-extended spinning particle. The results are either known or implicit in earlier work, but we were able to simply confirm both that the $\mathcal{N}=2$ quantum spinning particle has either spin zero or spin one, and that the $\mathcal{N}>2$ massive spinning particle is quantum inconsistent in even spacetime dimensions.

Implicit in our results for the massive spinning particle is a twistor description of the massless spinning particle for $D=3,4,6$, obtained by setting the mass to zero. One would expect to be able to simplify the action in this case so as to parametrize the bosonic phase space by the components of a single twistor, and we have spelt out the details of the procedure that achieves this for $D=3$. In all cases, the graded dimension of the physical phase space is the same as that found by taking the massless limit of the massive spinning particle in its standard phase space formulation, but this limit does not yield the usual massless spinning particle action: there remains an additional anticommuting 'spectator' variable. This discontinuity in the massless limit of the spinning particle appears not to have been commented on previously. 
In our twistor construction, we allowed for either sign of the energy when solving the mass-shell constraint on the $D$-momentum. A notable feature of our results for the twistorial action is that this sign choice does not appear in it. This does not happen for the superparticle, where the sign of the fermion kinetic terms is correlated with the sign of the energy, as pointed out in [27] in the context of a comparison of gauge-fixed superparticle and spinning particle actions. In an appendix we have confirmed this correlation for the massive 4D superparticle from a supertwistor form of its action constructed along the lines of the 6D case in [12].

Our results for massive spinning particles fit nicely with the association of the spacetime dimensions $D=3,4,6$ with the division algebras $\mathbb{R}, \mathbb{C}, \mathbb{H}$, and this suggests a possible octonionic extension to $D=10$. We expect the massive spinning particle to have a twistor formulation for which the bosonic variables are the components of a pair of octonionic twistors, i.e. two 4-plets of $\operatorname{Sp}(4 ; \mathbb{O})$. Appropriately defined [28], this is the $D=10$ conformal group. We also expect 22 spin-shell constraints in the form of an anti-hermitian $2 \times 2$ matrix over $\mathbb{O}$, and (from a reading of [28]) we would expect this to imply a $U(2 ; \mathbb{O}) \cong \operatorname{Spin}(9)$ gauge invariance (since $22+14=36$ ). If this is correct, and taking into account a mass-shell constraint, we would have a physical phase superspace of bosonic dimension $64-2(22+1)=18$. Given that the fermionic variables are again the entries of a traceless hermitian $2 \times 2$ matrix, now over $\mathbb{O}$ and presumably equivalent to a 9 of Spin(9), we would then have a physical phase superspace of graded dimension (18|9), as required.

\section{Acknowledgments}

We are grateful to Paul Howe for helpful correspondence, and to Alex Arvanitakis and Joaquim Gomis for discussions. LM acknowledges partial support from the National Science Foundation Award PHY-1214521. PKT acknowledges support from the UK Science and Technology Facilities Council (grant ST/L000385/1). AJR is supported by a grant from the London Mathematical Society, and he thanks the University of Groningen for hospitality during the writing of this paper. LM and PKT are grateful for the hospitality of the Pedro Pascual Benasque Center for Science, where part of this work was done.

\section{Appendix. Massive 4D superparticle}

In this appendix we present the twistor form of the minimal massive 4D superparticle in the notation of this paper, following the twistor construction of the $6 \mathrm{D}$ superparticle in [12]. The action is

$$
S=\int \mathrm{d} t\left\{-\frac{1}{2}\left(\dot{X}^{\alpha \dot{\alpha}}+\mathrm{i} \bar{\theta}^{\dot{\alpha}} \dot{\theta}^{\alpha}-\mathrm{i} \dot{\bar{\theta}}^{\dot{\alpha}} \theta^{\alpha}\right) P_{\alpha \dot{\alpha}}-\frac{1}{2} \mathrm{e}\left(P^{2}+m^{2}\right)\right\}
$$

Since the phase superspace has graded dimension (8|4) and there is one (first-class) constraint generating a gauge invariance, the physical phase superspace has graded dimension (6|4).

The action is manifestly invariant under the spacetime supersymmetry transformations

$$
\delta X^{\alpha \dot{\alpha}}=-\mathrm{i} \bar{\epsilon}^{\dot{\alpha}} \theta^{\alpha}-\mathrm{i} \epsilon^{\alpha} \bar{\theta}^{\dot{\alpha}}, \quad \delta \theta^{\alpha}=\epsilon^{\alpha} .
$$

The anticommuting Noether charges are

$$
Q_{\alpha}=P_{\alpha \dot{\alpha}} \bar{\theta}^{\dot{\alpha}}, \quad \bar{Q}_{\dot{\alpha}}=P_{\alpha \dot{\alpha}} \theta^{\alpha} .
$$


There are also hidden supersymmetry charges [26]:

$$
\tilde{Q}^{\alpha}=m \theta^{\alpha}, \quad \overline{\tilde{Q}}^{\dot{\alpha}}=m \bar{\theta}^{\dot{\alpha}} .
$$

The Poisson brackets of canonical variables follow directly from the action. The only non-zero one that we will need here is

$$
\left\{\theta^{\alpha}, \bar{\theta}^{\dot{\alpha}}\right\}_{\mathrm{PB}}=-\mathrm{i} P^{\alpha \dot{\alpha}} / P^{2} \text {. }
$$

Using this, the full algebra of supersymmetry charges, manifest and hidden, is found to be the BPS-saturated $\mathcal{N}=2$ supersymmetry algebra

$$
\begin{array}{ll}
\left\{Q_{\alpha}, \bar{Q}_{\dot{\alpha}}\right\}_{\mathrm{PB}}=\mathrm{i} P_{\alpha \dot{\alpha}}, & \left\{\tilde{Q}^{\alpha}, \overline{\tilde{Q}}^{\dot{\alpha}}\right\}_{\mathrm{PB}}=\mathrm{i} P^{\alpha \dot{\alpha}}, \\
\left\{Q_{\alpha}, \tilde{Q}^{\beta}\right\}_{\mathrm{PB}}=\mathrm{i} m \delta_{\alpha}^{\beta}, & \left\{\bar{Q}_{\dot{\alpha}}, \overline{\tilde{Q}}^{\dot{\beta}}\right\}_{\mathrm{PB}}=\mathrm{i} m \delta_{\dot{\alpha}}^{\dot{\beta}} .
\end{array}
$$

To pass to the supertwistor form of the action, we solve the mass-shell constraint as we did for the 4D spinning particle. Substitution for $P$ then yields the new Lagrangian

$$
L=\left[\dot{U}_{\alpha}^{I} W_{I}^{\alpha}+\dot{\bar{U}}_{\dot{\alpha} I} \bar{W}^{\dot{\alpha} I} \pm \mathrm{i} \bar{\mu}_{I} \dot{\mu}^{I}\right] \text {, }
$$

where

$$
\mu^{I}=\theta^{\alpha} U_{\alpha}^{I}, \quad \bar{\mu}_{I}=\bar{\theta}^{\dot{\alpha}} \bar{U}_{\dot{\alpha} I},
$$

and

$$
W_{I}^{\alpha}=\mp \frac{1}{2} X^{\dot{\alpha} \alpha} \bar{U}_{\dot{\alpha} I} \mp \frac{\mathrm{i}}{2} \bar{\mu}_{I} \theta^{\alpha}, \quad \bar{W}^{\dot{\alpha} I}=\mp \frac{1}{2} X^{\dot{\alpha} \alpha} U_{\alpha}^{I} \mp \frac{\mathrm{i}}{2} \mu^{I} \bar{\theta}^{\dot{\alpha}} .
$$

From these expressions we deduce the identity

$$
0 \equiv G_{J}^{I}:=U_{\alpha}^{I} W_{J}^{\alpha}-\bar{U}_{\dot{\alpha} J} \bar{W}^{\dot{\alpha} I} \mp \mathrm{i} \mu^{I} \bar{\mu}_{J} .
$$

This becomes a $U(2)$ constraint when we promote $W$ to an independent variable. This leads to the new action

$$
S=\int \mathrm{d} t\left\{U_{\alpha}^{I} \dot{W}_{I}^{\alpha}+\bar{U}_{\dot{\alpha} I} \bar{W}^{\dot{\alpha} I} \pm \mathrm{i} \bar{\mu}_{I} \dot{\mu}^{I}-s_{J}{ }^{I} G_{J}^{I}-\rho \varphi\right\},
$$

where $\varphi=|\operatorname{det} U|^{2}-m^{2}$, as for the massive spinning particle.

As a check on this action, one may verify that the (graded) dimension of the physical phase superspace is still (6|4). The $S U(2)$ doublet of twistors $(U, W)$ have $2 \times 4=8$ complex components, giving a real bosonic dimension of 16 , but there are four spin-shell constraints, with spin-shell algebra $U(2)$, and one further mass-shell-type constraint; all are first class so the physical bosonic dimension of $16-2 \times 5=6$. The new anticommuting variable $\mu^{I}$ is a complex doublet of $U(2)$, and there are no fermionic constraints, so the physical fermionic dimension is 4.

A crucial feature of this superparticle action, which is required by spacetime supersymmetry [27], is the correlation between the sign of the energy and the sign of the 'fermionic kinetic term'.

\section{References}

[1] Nahm W 1978 Supersymmetries and their representations Nucl. Phys. B 135149

[2] Brink L and Schwarz J H 1981 Quantum superspace Phys. Lett. B 100310 
[3] Siegel W 1983 Hidden local supersymmetry in the supersymmetric particle action Phys. Lett. B 128397

[4] Shirafuji T 1983 Lagrangian mechanics of massless particles with spin Prog. Theor. Phys. 7018

[5] Bengtsson I and Cederwall M 1988 Particles, twistors and the division algebras Nucl. Phys. B 30281

[6] Ferber A 1978 Supertwistors and conformal supersymmetry Nucl. Phys. B 13255

[7] Casalbuoni R 1976 The classical mechanics for Bose-Fermi systems Nuovo Cimento A 33389

[8] Hughston L P and Hurd T R 1981 A cohomological description of massive fields Proc. R. Soc. A 378141

[9] Penrose R and Rindler W 1988 Spinors and Space-Time. Volume 2. Spinor And Twistor Methods In Space-Time Geometry (Cambridge: Cambridge University Press)

[10] de Azcárraga J A, Izquierdo J M and Lukierski J 2009 Supertwistors, massive superparticles and k-symmetry J. High Energy Phys. JHEP01(2009)041

[11] Mezincescu L, Routh A J and Townsend P K 2014 Supertwistors and massive particles Ann. Phys. 34666

[12] Routh A J and Townsend P K 2015 Twistor form of massive 6D superparticle arXiv:1507.05218 [hep-th]

[13] Kugo T and Townsend P K 1983 Supersymmetry and the division algebras Nucl. Phys. B 221357

[14] Sudbery A 1984 Division algebras, (pseudo)orthogonal groups and spinors J. Phys. A: Math. Gen 17939

[15] Townsend P K 1991 Supertwistor formulation of the spinning particle Phys. Lett. B 26165

[16] Brink L, Deser S, Zumino B, Di Vecchia P and Howe P S 1976 Local supersymmetry for spinning particles Phys. Lett. B 64435

Brink L, Deser S, Zumino B, Di Vecchia P and Howe P S 1977 Phys. Lett. B 68488 (erratum)

[17] Brink L, Di Vecchia P and Howe P S 1977 A Lagrangian formulation of the classical and quantum dynamics of spinning particles Nucl. Phys. B 11876

[18] Gershun V D and Tkach V I 1979 Classical and quantum dynamics of particles with arbitrary spin JETP Lett. 29288

Gershun V D and Tkach V I 1979 Pisma Zh. Eksp. Teor. Fiz. 29320

[19] Howe P S, Penati S, Pernici M and Townsend P K 1988 Wave equations for arbitrary spin from quantization of the extended supersymmetric spinning particle Phys. Lett. B 215555

[20] Howe P S, Penati S, Pernici M and Townsend P K 1989 A particle mechanics description of antisymmetric tensor fields Class. Quantum Grav. 61125

[21] Shaw W T 1985 Twistor, minimal surfaces and strings Class. Quantum Grav. 2 L113

[22] Cederwall M 1989 An extension of the twistor concept to string theory Phys. Lett. B 22645

[23] Howe P S 1982 Supergravity in superspace Nucl. Phys. B 199309

[24] Elitzur S, Frishman Y, Rabinovici E and Schwimmer A 1986 Origins of global anomalies in quantum mechanics Nucl. Phys. B 27393

[25] Howe P S, Sierra G and Townsend P K 1983 Supersymmetry in six-dimensions Nucl. Phys. B 221331

[26] Mezincescu L, Routh A J and Townsend P K 2014 All superparticles are BPS J. Phys. A 47 175401

[27] Gauntlett J P, Gomis J and Townsend P K 1990 Supersymmetry and the physical phase space formulation of spinning particles Phys. Lett. B 248288

[28] Chung K W and Sudbery A 1987 Octonions and the Lorentz and conformal groups of tendimensional space-time Phys. Lett. B 198161 